\title{
POSSIBILIDADES E LIMITAÇÕES: COMPARTILHANDO EXPERIÊNCIAS VIVENCIADAS NO ESTÁGIO SUPERVISIONADO EM ENFERMAGEM
}

\author{
Thaisy Sarmento Batista de OLIVEIRA ${ }^{1}$ \\ Helloysa Karynna Sttefanny de Oliveira E SILVA ${ }^{2}$ \\ José Barros FILHO 3 \\ Priscila Raquel Dantas SOARES ${ }^{4}$ \\ Sybele Oliveira de SOUZA ${ }^{5}$
}

\begin{abstract}
${ }^{1}$ Professora no Curso de Bacharelado em Enfermagem na Universidade Federal de Campina Grande - UFCG, campus
Cuité - PB. E-mail: thaisysarmento@hotmail.com

${ }^{2}$ Graduanda no Curso de Bacharelado em Enfermagem na Universidade Federal de Campina Grande - UFCG, campus Cuité - PB. E-mail: karynna2007@bol.com.br

${ }^{3}$ Graduando no Curso de Bacharelado em Enfermagem na Universidade Federal de Campina Grande - UFCG, campus Cuité - PB. E-mail: zeckbarros@hotmail.com

4 Graduanda no Curso de Bacharelado em Enfermagem na Universidade Federal de Campina Grande - UFCG, campus Cuité - PB. E-mail: priscila.soares.enf@hotmail.com

5 Graduanda no Curso de Bacharelado em Enfermagem na Universidade Federal de Campina Grande - UFCG, campus Cuité - PB. E-mail: sybeleufcghotmail.com
\end{abstract}

Recebido em: 19/02/2014 - Aprovado em: 30/06/2014 - Disponibilizado em: 30/07/2014

\section{RESUMO}

Introdução: A vivência do estágio supervisionado proporciona ao acadêmico de enfermagem o desenvolvimento de diferentes habilidades e competências imprescindíveis à sua formação. Objetivo: Compartilhar as experiências vividas na clínica médica masculina, visto ter sido uma ocasião de amplo incremento aos alunos a vivência nesse serviço. Metodologia: Trata-se de um relato de experiência, vivenciado por discentes do Curso de Bacharelado em Enfermagem da Universidade Federal de Campina Grande - Campus Cuité - PB durante o Estágio Supervisionado em Enfermagem II no Hospital Universitário Alcides Carneiro - HUAC, no $10^{\circ}$ período do curso, onde os alunos estagiaram em média 10 turnos, no período entre Outubro de 2013 e Janeiro de 2014, e sob a supervisão direta da enfermeira do setor. Resultados e Discussão: O período de estágio na clínica médica masculina proporcionou aos estudantes grande aprendizagem e crescimento para seu futuro profissional, onde puderam perpetrar uma diversidade de conhecimentos provindos de toda carga teórica do curso e até mesmo o que nunca foi visto na graduação, pois o fluxo das demandas na enfermagem favorece projeções de conhecimentos tenros, quer seja com profissionais, quer seja com pacientes, havendo uma continuidade do saber. Considerações Finais: Concluise que a experiência foi válida, evidenciada pelo aprendizado e maior autoconfiança adquirida, contribuindo para uma qualificada formação profissional, fazendo com que a inserção futura para o mercado de trabalho flua com mais segurança e a prestação da assistência seja humanizada e de qualidade.

Palavras-chave: Estágio Clínico. Supervisão de Enfermagem. Estudantes de Enfermagem. Cuidados de Enfermagem. Serviço Hospitalar de Admissão de Pacientes.

\section{ABSTRACT}

Introduction: The experience of supervised internship provides the nursing students develop different skills and competencies essential to their formation. Objective: To share the experiences in male medical clinic, since it was an opportunity for students to increase broad experience in this service. Methodology: This is an experience report, experienced by students of the Bachelor of Nursing, Federal University of Campina 
Grande - Campus Cuité - PB during Supervised Nursing II at University Hospital Alcides Carneiro - HUAC, the 10th period course, where students aged in average 10 shifts, between October 2013 and January 2014, and under the direct supervision of the nurse in the sector. Results and Discussion: The probationary period in the male medical clinic has provided great student learning and growth for their future careers, where they could perpetrate a diversity of knowledge stemmed from all theoretical course load and even that was never seen in the graduation because the flow of demands in nursing favors projections tender knowledge, whether with professionals, whether with patients, with a continuity of knowledge. Final Thoughts: It is concluded that the experience was valid, as evidenced by learning and increased confidence gained, contributing to a qualified vocational training, making the future insertion into the labor market to flow more safely and delivery of assistance is humanized and quality.

Keywords: Clinical Clerkship. Nursing, Supervisory. Students, Nursing. Nursing Care. Admitting Department, Hospital.

\section{INTRODUÇÃO}

De acordo com o disposto no art.7 da resolução do CNE/CES $\mathrm{N}^{\circ} 3$, de 7 de novembro de 2001, que institui diretrizes curriculares nacionais dos cursos de graduação em enfermagem, o fluxograma do curso, as instituições ficam obrigadas a incluir no currículo o estágio supervisionado em enfermagem no último ano do curso, possibilitando aos alunos vivenciarem na prática, maior parte dos conteúdos vistos no decorrer da graduação, promovendo sua capacidade de desenvolvimento nas diversas atribuições enquanto enfermeiro.

$\begin{array}{cccr}\text { Nesse } & \text { sentido, } & \text { o } & \begin{array}{r}\text { estágio } \\ \text { supervisionado } \\ \text { torna-se }\end{array} \\ \text { importante na } & \text { formação } & \text { profissional do do }\end{array}$ enfermeiro, pois, para além das considerações salientadas na resolução $\mathrm{N}^{0} 3$, do CNS/CES acerca da vivência do estágio supervisionado, destaca-se esse momento, como uma oportunidade para superar possíveis déficits nas atividades práticas ocorridas durante todo curso.
A vivência do estágio supervisionado proporciona ao acadêmico de enfermagem o desenvolvimento de diferentes habilidades e competências imprescindíveis à sua formação. O contexto do trabalho estimula o desenvolvimento da autonomia, responsabilidade, liberdade, criatividade, compromisso, domínio da prática e de seu papel social, aprofundamento e contextualização dos conhecimentos, assumindo uma práxis transformadora. (BENITO et al, 2012)

O Hospital escola dos alunos do curso de enfermagem da Universidade Federal de Campina Grande, campus Cuité, é o Hospital Universitário Alcides Carneiro (HUAC), situado no município de Campina Grande/PB, onde os alunos no $10^{\circ}$ período da graduação vivenciam o estágio supervisionado supracitado, tendo que cumprir uma carga horária mínima de 405 horas.

Para realização desse relato, foi selecionado um dos setores que os discentes vivenciaram na prática, e o de escolha foi a clínica médica masculina, onde os alunos 
estagiaram em média 10 turnos e sob a supervisão direta da enfermeira do setor, prestaram assistência de enfermagem aos pacientes internados, bem como realizaram tarefas gerenciais que compete ao enfermeiro.

A clínica médica masculina do HUAC atende pacientes adultos com doenças crônicas não-transmissíveis, que demandam cuidados de equipes multiprofissionais. Tratase de um setor dinâmico, com constantes atividades: admissões, realização de exames físico, coleta de exames laboratoriais, realização de procedimentos invasivos, curativos, entre outros. Os pacientes deste setor, fazem uso de dispositivos como: sonda vesical de demora e/ou de alívio, acesso venoso periférico e/ou central, colostomias, drenos, curativos, entre outros, sendo de suma proficiência para os alunos, conferindo oportunidade aos mesmos de realizações de procedimentos técnicos, gerenciais, fato no qual tornou o principal ponto positivo da vivência dos estudantes.

Diante do exposto, o objetivo desse estudo é compartilhar as experiências vividas no estágio supervisionado com enfoque no setor supracitado, visto ter sido uma ocasião de amplo incremento aos alunos a vivência nesse serviço, a despeito do breve período de estágio no referido setor, foi um período suficiente para tornar conhecida a rotina do setor, assim como colocar em prática a pluralidade dos ensinamentos obtidos na teoria.

\section{METODOLOGIA}

Trata-se de um relato de experiência, vivenciado por discentes do Curso de Bacharelado em Enfermagem da Universidade Federal de Campina Grande Campus Cuité - PB durante o Estágio Supervisionado em Enfermagem II no Hospital Universitário Alcides Carneiro HUAC, no $10^{\circ}$ período do curso, onde os alunos estagiaram em média 10 turnos, no período entre Outubro de 2013 e Janeiro de 2014, e sob a supervisão direta da enfermeira do setor.

A clínica médica masculina (ALA D) possui 6 enfermarias, cada uma com um banheiro, sendo uma com leito isolado, e as demais contendo em média de 3 à 6 leitos, totalizando 19 leitos neste setor. Possui ainda expurgo, posto de enfermagem, copa, sala de prescrição médica e repouso da enfermagem. O setor possui 1 enfermeiro diarista em cada plantão, com em média 4 técnicos, 1 enfermeiro plantonista, com em média 3 técnicos.

Durante o período de estágio foram desenvolvidas atividades como: a evolução de enfermagem, realização de procedimentos invasivos, realização de curativos, entre outros. 


\section{RESULTADOS E DISCUSSÃO}

\section{Identificação do setor}

Se tratando dos recursos físicos, o setor possui 6 enfermarias, cada uma com um banheiro para os pacientes e seus acompanhantes, sendo uma com um leito isolado e as demais contendo em média de 3 à 6 leitos, totalizando 19 leitos neste setor. Dispõe ainda de expurgo para desprezar materiais contaminados, posto de enfermagem, copa, sala de prescrição médica e repouso da enfermagem.

Com relação aos recursos humanos, é assumido no setor em cada plantão 1 enfermeiro diarista, com 4 técnicos em média, 1 enfermeiro plantonista, com cerca de 3 técnicos.

No que concerne os recursos materiais, observou-se que no posto de enfermagem contém materiais necessários para realização de procedimentos rotineiros, armários para armazenamentos de roupas e lençóis para pacientes, duas pias para lavagem das mãos, geladeira, bebedouro e armário para armazenar impressos.

As enfermarias possuem macas modernas, oferecendo maior conforto e praticidade aos pacientes, poltronas para os acompanhantes, banheiro, algumas detém ainda mesa para auxiliar na realização de procedimentos.

\section{Descrição das atividades desenvolvidas no} setor

A clínica médica masculina do HUAC é bastante dinâmica, por conseguinte, os profissionais e estagiários continuamente encontram-se em exercício da assistência de enfermagem e serviços burocráticos do setor, dessa forma, o setor torna-se cenário profícuo para os estagiários de enfermagem no que concerne à obtenção de prática e experiência.

No tocante às atividades desenvolvidas, foi prestada pelos estagiários assistência direta ao paciente, organização do serviço, e anotações de enfermagem. Entre os procedimentos realizados, podemos citar: exame físico e evolução de enfermagem dos pacientes que nos proporcionou acompanhar de perto a evolução do quadro clínico de cada paciente, os sinais e sintomas de suas patologias assim como o tratamento oferecido e a reação dos mesmos quanto as condições e exigências que cada tratamento requer; organização de materiais no posto de enfermagem onde nos mostrou que a organização melhora a execução das atividades assim como diminui a probabilidades de erros; coleta de sangue arterial para gasometria, HGT, aplicação de insulina, preparo e administração de medicamentos, curativos, aspiração endotraqueal, todos esses procedimentos nos proporcionaram vivenciar a realização e aplicação das técnicas necessárias assim como treinarmos nossas habilidades para realização 
de tais procedimentos; ainda fizemos o controle dos antibióticos, acompanhamento dos pacientes para realização de exames como: RX, tomografia computadorizada, eletrocardiograma; relatamos as ocorrências no final de cada plantão no livro de ocorrências assim como presenciamos a passagem do plantão pro enfermeiro que ia assumir o setor, e por fim, quando algum paciente era admitido ou saia de alta nós preenchíamos o livro do relatório geral.

Além das atividades citadas, foram adotadas ações que promoveram maior conforto físico e psicológico aos pacientes, como: esclarecimento de suas dúvidas a respeito do seu estado de saúde, orientações de autocuidado, medidas de conforto, mudanças de decúbito em pacientes debilitados ao leito, com intenção de prevenir úlceras por pressão, e apoio emocional.

Todas as atividades realizadas neste setor, seja ela gerencial ou assistencial, foram ímpar, pois durante o curso não tínhamos tido a oportunidade de vivenciar a realidade de ser enfermeiro e essa prática nos proporcionou aprendizado profissional e pessoal, conhecimentos e ensinamentos que nos prepara para vida fora da academia sem a supervisão de alguém responsável por nós.

\section{Relato do período de experiência, incluindo facilidades e dificuldades vivenciadas}

O período de estágio na clínica médica masculina proporcionou aos estudantes grande aprendizagem e crescimento para seu futuro profissional, onde puderam perpetrar uma diversidade de conhecimentos provindos de toda carga teórica do curso e até mesmo o que nunca foi visto na graduação, pois o fluxo das demandas na enfermagem favorece projeções de conhecimentos tenros, quer seja com profissionais, quer seja com pacientes, havendo uma continuidade do saber.

Corroborando com o exposto, Mattosinho et al (2010) relata que o preparo da graduação é só o começo, pois o profissional deve estar em constante aprendizado, buscando o desenvolvimento da habilidade, dos conhecimentos, das atitudes e da experiência necessária na qualificação do enfermeiro. Assim, a passagem para o mercado de trabalho torna-se prazerosa, gratificante e instigante, estimulando o profissional a superar os desafios e os limites de sua formação profissional.

Entre as facilidades desse período de estágio, pode-se citar a receptividade dos enfermeiros para com os alunos, concedendoos oportunidades e ensinamentos; o acolhimento dos pacientes, permitindo a realização de procedimentos, transmitindo total confiança. Dessa forma, os alunos apresentaram uma melhor desenvoltura na realização dos procedimentos, fato no qual os propiciou maior interesse, gerando motivação e conferindo um estágio produtivo.

Porém, houve algumas exceções, no que concerne ao acolhimento de um dos 
profissionais. Sabe-se que um dos itens fundamentais para o sucesso de uma profissão é o relacionamento interpessoal harmonioso, no entanto, neste setor foi vivenciada por um dos alunos uma má receptividade por parte de uma profissional, implicando numa situação frustrante para a aluna, o que tornou um ponto negativo do estágio para a mesma.

Foi observado também, que no setor em destaque, não há implementação da sistematização da assistência de enfermagem (SAE), uma vez que este é uma ferramenta de fundamental importância para o enfermeiro realizar uma assistência de enfermagem de qualidade.

A SAE representa a forma como o trabalho da enfermagem é organizado, de acordo com o método científico e o referencial teórico, de modo que seja possível o melhor atendimento das necessidades de cuidado do cliente, família e comunidade pela aplicação das fases que compõem o processo de enfermagem, sendo elas: histórico de enfermagem, diagnóstico de enfermagem, planejamento, implementação e avaliação.

De acordo com a resolução Resolução COFEN n ${ }^{\circ} 358 / 2009$, a SAE e o Processo de Enfermagem representam uma necessidade colocada cada vez mais frequentemente pelos serviços de saúde. O desenvolvimento de competências para sua implementação de modo planejado e dinâmico, permite identificar, compreender, descrever, explicar e predizer quais as necessidades do indivíduo, família ou coletividade humana, em determinado momento do processo saúde e doença. (MALUCELLI et al, 2010.)

\section{Análise crítica da vivência}

O Estágio Supervisionado II foi de alta relevância, oportunizando maior contato com o paciente durante seu diagnóstico, tratamento da patologia e recuperação, principalmente, à realidade de um hospital na sua dinâmica diária onde se trata de um lugar de fácil acesso ao aprendizado não meramente por ser um hospital escola, mas pela sua extensa variedade de serviços, demanda e pelo acolhimento dos profissionais.

Durante o período de estágio, estabeleceu-se uma boa relação com a equipe de enfermagem, com algumas exceções, contribuindo para obtenção de um significativo apoio que proporcionou o aprendizado dos alunos.

A avaliação acerca da disciplina em junção com o serviço apresentado à população é considerada estável, pois podemos perceber a real necessidade do Estágio Supervisionado em Enfermagem II na grade curricular do curso de bacharelado em enfermagem. Essa avaliação nos fornece um entendimento de como é prestado os serviços assistenciais à população, e uma avaliação expletiva da estrutura que consideramos boa para o funcionamento do HUAC, e dos profissionais 
atuantes, se mostrando capacitados para postularem os referidos cargos.

É compreensível que poderia haver um maior êxito, porém, ainda assim foi possível relacionar as atividades práticas com a teoria absorvida durante o curso, como também se identificou a importância dessa associação.

\section{CONSIDERAÇÕES FINAIS}

O estágio curricular supervisionado no HUAC foi de ampla importância para os acadêmicos. De modo particular a clínica médica masculina, foi uma escola para os estudantes, pelo fato de existirem oportunidades diversas de realização tanto de procedimentos técnicos, quanto de atividades gerenciais, considerando os déficits acumulados no decorrer do curso.

O estágio tornou-se ainda de grande valia, pelo fato do setor citado abranger um repertório de patologias, inclusive raras, possibilitando aos estudantes tocar nos conhecimentos recém-adquiridos, bem como aprofundar os pré-existentes. Salientando que a confiança dos pacientes para com os alunos, motivou na realização das atividades práticas durante o estágio.

No que se refere à organização do estágio, sugere-se um melhor planejamento antecipado da disciplina em questão, que acarretará melhorias relacionadas ao campo de estágio, estadia dos alunos e condições necessárias para estudo e pesquisa, maior abrangência de áreas assistenciais a serem vistas por todos os alunos igualmente, dentre outras, que um adequado planejamento minimizará as deficiências enfrentadas no estágio supervisionado em enfermagem II.

Com relação à clínica médica masculina do HUAC, é oportuno sugerir a implementação efetiva da SAE, visto que é um instrumento fundamental para a oferta de uma assistência digna e holística, e com a aplicação deste, não há dúvidas que irá melhorar a qualidade de vida dos pacientes internos no setor citado.

É importante ressaltar ainda, que diante das dificuldades pessoais durante o estágio, tornou-se possível refletir sobre a relevância do companheirismo e do trabalho em equipe, pois é perceptível o melhor desempenho num setor, em vista da existência de um vínculo em que há contribuição e apoio, propiciando uma inclinação para o sucesso das atividades.

Dessa forma, conclui-se que a experiência foi válida, evidenciada pelo aprendizado e maior autoconfiança adquirida, contribuindo para uma qualificada formação profissional, fazendo com que a inserção futura para o mercado de trabalho flua com mais segurança e a prestação da assistência seja humanizada e de qualidade. 


\section{REFERÊNCIAS}

BENITO, G. A. V. et al. Desenvolvimento de competências gerais durante o estágio supervisionado. Rev Bras Enferm, Brasília, 65(1): 172-8, jan-fev, 2012. Disponível em: $<$ http://www.scielo.br/pdf/reben/v65n1/25.pdf > Acesso em: 28 jan. 2014.

MALLUCELLI, A. et al. Sistema de informação para apoio à Sistematização da Assistência de Enfermagem. Rev Bras

Enferm, Brasília, 63(4): 629-36, jul-ago, 2010. Disponível em: < http://www.scielo.br/pdf/reben/v63n4/20.pdf> Acesso em: 06 fev. 2014.

MATTOSINHO, M. M. S. et al. Mundo do trabalho: alguns aspectos vivenciados pelos profissionais recém- formados em enfermagem. Acta Paul Enferm, Florianopolis, 23(4):466-71, 2010. Disponível em:

$<$ http://www.scielo.br/scielo.php?script=sci_a rttext\&pid=S0103-21002010000400004> Acesso em: 28 jan. 2014.

\section{RESOLUÇÃO CNE/CES Nº 3, DE 7 DE}

NOVEMBRO DE 2001. Disponível em:

$<$ http://portal.mec.gov.br/cne/arquivos/pdf/CE S03.pdf> Acesso em: 14 fev. 2014.

RESOLUÇÃO COFEN No 358 DE 23 DE

OUTUBRO DE 2009. Disponível em: < http://novo.portalcofen.gov.br/resoluo-cofen2722002-revogada-pela-resoluao-cofen-n3582009_4309.html> Acesso em: 06 fev. 2014. 